\title{
Phenomenological application of the projection-operator method and its connection with critical dynamics
}

\author{
Edson Sardella* \\ Universidade Estadual Paulista Julio de Mesquita Filho, Campus de Ilha Solteira, Ilha Solteira-Săo Paulo, CEP 15378, Brazil
}

(Received 8 May 1990; revised manuscript received 25 June 1990)

\begin{abstract}
We consider the dynamics of a system of interacting spins described by the Ginzburg-Landau Hamiltonian. The method used is Zwanzig's version of the projection-operator method, in contrast to previous derivations in which we used Mori's version of this method. It is proved that both methods produce the same answer for the Green's function. We also make contact between the projection-operator method and critical dynamics.
\end{abstract}

MS code no. BS4191 1990 PACS number(s): 05.90. $+\mathrm{m}, 64.60 . \mathrm{Ht}$

It is well known that the average of any physical quantity at time $t$ can be evaluated in two different ways: first, by following the evolution of the physical variables and taking the distribution function time independent (the equilibrium distribution function), or by following the evolution of the distribution function and taking the physical variables time independent. These two ways of studying a physical system consist of solving an equation of motion of a dynamical variable or a Liouville equation for the distribution function, respectively. By introducing a projection-operator method (POM), Mori ${ }^{1}$ and Zwanzig ${ }^{2}$ showed that both equations can be rewritten in terms of a generalized Langevin equation and a generalized master equation, respectively. ${ }^{3}$ In a series of two papers $^{4,5}$ (paper I and paper II), we applied the Mori method to a phenomenological model of a system of interacting spins described by the Ginzburg-Landau (GL) Hamiltonian. The Green's function and the self-energy were calculated with this alternative approach instead of the standard Feynman diagram method. In this paper we will investigate the same system by starting from the generalized master equation, and it will be proved that this produces the same results as those found in paper I. We will also discuss the (slight) difference between our results and those from previous works, ${ }^{6}$ and we anticipate that they are not important from the point of view of critical dynamics when the system approaches the critical point. Therefore, the major aims of this paper are to make a contact between the POM and stochastic ideas of the critical dynamics phenomena, and to demonstrate an equivalence between both versions of the POM when it is applied to the system specified above.

Let us consider the dynamical evolution of a system of interacting spins. For simplicity, we will not regard interaction between the spin modes and any other sort of relaxation mode (for example, heat diffusion mode). We will also neglect the effects of the fast modes, i.e., we assume that the relaxation time of the fast modes are much shorter than the relaxation time of the corresponding slow modes. In this case, the Fokker-Planck equation of the system under consideration is ${ }^{6(a)}$

$$
\begin{gathered}
\frac{\partial \rho}{\partial t}=\gamma \sum_{\mathbf{k}_{1}}\left[\frac{\partial}{\partial \sigma\left(\mathbf{k}_{1}\right)} \frac{\partial H}{\partial \sigma\left(-\mathbf{k}_{1}\right)}+\frac{\partial H}{\partial \sigma\left(\mathbf{k}_{1}\right)} \frac{\partial}{\partial \sigma\left(-\mathbf{k}_{1}\right)}\right. \\
\left.+\frac{\partial}{\partial \sigma\left(\mathbf{k}_{1}\right)} \frac{\partial}{\partial \sigma\left(-\mathbf{k}_{1}\right)}\right] \rho,
\end{gathered}
$$

where $\rho\left(\left\{\sigma\left(\mathbf{k}_{i}\right)\right\}, t\right)$ is the distribution function, $\gamma$ is the spin diffusion coefficient, and $\sigma(\mathbf{k})$ is the spatial Fourier transform of the local spin density $\sigma(\mathbf{r})$. Here, $H$ is the GL Hamiltonian:

$$
\begin{aligned}
H=\frac{1}{2} & \sum_{\mathbf{k}_{1}}\left(r+k_{1}^{2}\right) \sigma\left(\mathbf{k}_{1}\right) \sigma\left(-\mathbf{k}_{1}\right) \\
+\frac{u}{8 V} \sum_{\mathbf{k}_{1}, \mathbf{k}_{2},} & \sigma\left(\mathbf{k}_{1}\right) \sigma\left(\mathbf{k}_{2}\right) \sigma\left(\mathbf{k}_{3}\right) \sigma\left(\mathbf{k}_{4}\right) \\
\mathbf{k}_{3}, \mathbf{k}_{4} & \\
& \times \Delta\left(\mathbf{k}_{1}+\mathbf{k}_{2}+\mathbf{k}_{3}+\mathbf{k}_{4}\right),
\end{aligned}
$$

where $r$ and $u$ are both phenomenological constants, $V$ is the volume of the system, and $\Delta$ is the Kronecker delta.

We can also write Eq. (1) as

$$
\frac{\partial \rho}{\partial t}=D \rho
$$

where $D$ is the Fokker-Planck operator:

$$
\begin{aligned}
D=\gamma \sum_{\mathbf{k}_{1}} & \left(\frac{\partial}{\partial \sigma\left(\mathbf{k}_{1}\right)} \frac{\partial H}{\partial \sigma\left(-\mathbf{k}_{1}\right)}+\frac{\partial H}{\partial \sigma\left(\mathbf{k}_{1}\right)} \frac{\partial}{\partial \sigma\left(-\mathbf{k}_{1}\right)}\right. \\
& \left.+\frac{\partial}{\partial \sigma\left(\mathbf{k}_{1}\right)} \frac{\partial}{\partial \sigma\left(-\mathbf{k}_{1}\right)}\right) .
\end{aligned}
$$

Now, the master equation associated with Eq. (3) is ${ }^{7}$

$$
\frac{\partial}{\partial t} P \rho(t)=P D P \rho(t)-\int_{0}^{t} d \tau K(\tau) P \rho(t-\tau),
$$

where the kernel (operator) is

$$
K(t)=-P D(1-P) e^{(1-P) D \tau}(1-P) D P,
$$


and $P$ is the projection operator defined below. In Eq. (5) we have used the abbreviated notation $\rho(t)$ $=\rho\left(\left\{\sigma\left(\mathbf{k}_{i}\right)\right\}, t\right)$. In deriving Eq. (5), we have employed the initial condition $P \rho(0)=\rho(0)$. We will make a special choice, so that this condition is satisfied. As in Ref. 7, we define $P$ as

$$
P G=\rho_{0} \int d \Gamma G+\frac{\sigma(-\mathbf{k}) \rho_{0}}{\left\langle|\sigma(\mathbf{k})|^{2}\right\rangle_{0}} \int d \Gamma G \sigma(\mathbf{k}),
$$

where $G$ is an arbitrary function of $\left(\left\{\sigma\left(\mathbf{k}_{i}\right)\right\}, t\right)$; $\rho_{0}=e^{-H} / \operatorname{Tr}\left(e^{-H}\right)$ is the stationary solution of Eq. (3); $d \Gamma=\Pi_{k} d \sigma(\mathbf{k})$, and the integration is defined by taking the integral over their real and imaginary parts. The equilibrium distribution function $\rho_{0}$ is used to take the equilibrium averages here denoted by $\langle\cdots\rangle_{0}$. [Those interested in the motivations which lead to the definition of the projection operator, as in Eq. (7), should consult Ref. 7. However, it must be noted that some changes were made in order to adapt to the present situation, in which the fast modes have been neglected. We must also make another remark with respect to the definition of $\rho_{0}$. Note that, without loss of generality, we have taken $\left.K_{B} T=1 .^{6(\mathrm{a})}\right]$

Let us move our discussion to the derivation of the correlation function of $\sigma$. This function is usually defined by

$$
C(\mathbf{k}, t)=\langle\sigma(\mathbf{k}, t) \sigma(-\mathbf{k})\rangle_{0},
$$

where the average is taken over the equilibrium distribution function, and the Fourier component of local spin density at time $t$ is given by the Langevin equation: ${ }^{6(a)}$

$$
\frac{d}{d t} \sigma(\mathbf{k}, t)=-\gamma \frac{\partial H}{\partial \sigma(-\mathbf{k})}+\mathscr{F}(t),
$$

where $\mathcal{F}(t)$ is a randomly fluctuating force. It was pointed out by Bixon and $\mathrm{Zwanzig}^{7}$ that if we average first over the randomly fluctuating force, then the Liouville operator associated with the Langevin equation of motion is replaced by the Fokker-Planck operator given by Eq. (4), and the correlation function is given by

$$
C(\mathbf{k}, t)=\int d \Gamma \sigma(\mathbf{k}) e^{D \tau} \sigma(-\mathbf{k}) \rho_{0}
$$

Now, if we take $G=\rho$ in Eq. (7), multiply both sides by $\sigma(\mathbf{k})$, and then integrate over the phase space, we obtain

$$
\begin{aligned}
\int d \Gamma \sigma(\mathbf{k}) P \rho(t)= & \int d \Gamma \sigma(\mathbf{k}) \\
& \times \int \rho_{0} \int d \Gamma \rho(t) \\
& \left.\quad+\frac{\sigma(-\mathbf{k}) \rho_{0}}{\left\langle|\sigma(\mathbf{k})|^{2}\right\rangle_{0}} \int d \Gamma \rho(t) \sigma(\mathbf{k})\right] \\
= & \langle\sigma(\mathbf{k})\rangle_{0} \int d \Gamma \rho(t) \\
& +\int d \Gamma \rho(t) \sigma(\mathbf{k}) \\
= & \int d \Gamma \rho(t) \sigma(\mathbf{k}),
\end{aligned}
$$

where we have used the fact that $\langle\sigma(\mathbf{k})\rangle_{0}=0$, which is valid only above the critical temperature.

It can be easily seen that the formal solution of Eq. (3) is $\rho(t)=e^{D t} \rho(0)$. Therefore, if we take the initial condition, $\rho(0)=\sigma(-\mathbf{k}) \rho_{0}$, a direct comparison between Eqs. (10) and (11) shows us that the projected part of $\rho$ correctly gives the correlation function $C(\mathbf{k}, t)$. It is in this sense that $\mathrm{Zwanzig}^{7}$ calls $P \rho$ the relevant part of $\rho$. Note that this initial condition obeys $P \rho(0)=\rho(0)$, as required in the derivation of Eq. (5).

In order to find an equation for the correlation function, we must multiply both sides of Eq. (5) by $\sigma(\mathbf{k})$ and then integrate over the phase space. In Appendix A, we then find that

$$
\begin{aligned}
& \int d \Gamma[P D P \rho(t)] \sigma(\mathbf{k})=-\omega(\mathbf{k}) C(\mathbf{k}, t), \\
& \int d \Gamma[K(\tau) P \rho(t-\tau)] \sigma(\mathbf{k})=\Phi(\mathbf{k}, \tau) C(\mathbf{k}, t-\tau),
\end{aligned}
$$

where

$$
\begin{aligned}
& \omega(\mathbf{k})=\gamma /\left\langle|\sigma(\mathbf{k})|^{2}\right\rangle_{0}, \\
& \Phi(\mathbf{K}, t)=\int d \Gamma f(-\mathbf{k}) f(\mathbf{k}, t), \\
& f(\mathbf{k}, t)=\gamma e^{(1-P) D t}\left(\frac{\sigma(-\mathbf{k}) \rho_{0}}{\left\langle|\sigma(\mathbf{k})|^{2}\right\rangle_{0}}+\rho_{0} \frac{\partial H}{\partial \sigma(-\mathbf{k})}\right) .
\end{aligned}
$$

Using Eq. (5) with the help of Eqs. (12) and (13), we finally obtain for the correlation function the following:

$$
\frac{d}{d t} C(\mathbf{k}, t)=-\omega(\mathbf{k}) C(\mathbf{k}, t)-\int_{0}^{t} d \tau \Phi(\mathbf{k}, \tau) C(\mathbf{k}, t-\tau)
$$

This is precisely what we found in paper I by using the Mori method for a monovariate process, with the dynamical variable given by $A(\mathbf{k}, t)=e^{i L t} A(\mathbf{k}), A(\mathbf{k})=\sigma(\mathbf{k}) \rho_{0}$, and $i L=D$. However, for this end we had to make a rather unusual definition of the scalar product of the dynamical variables, but this produces the correct definition of the correlation function.

Although Eq. (17) is linear in $C(\mathbf{k}, t)$, we cannot solve it exactly, because the memory function $\Phi(\mathbf{k}, t)$ cannot be evaluated explicitly for all values of $u$. This is because $f(\mathbf{k}, t)$ is governed by the unusual propagator $e^{(1-P) D t}$ instead of $e^{D t}$. Nevertheless, if we treat the second term of Eq. (2) as a small perturbation of the GL Hamiltonian (with $u$ being the perturbative parameter), it is possible to evaluate the memory function to second order in $u$. This calculation and the calculation of the Green's function and the self-energy have been considered elsewhere (paper I), but, for the sake of completeness, we will quote the results, respectively, ${ }^{8}$ as 


$$
\begin{aligned}
& \Phi(\mathbf{k}, t)=\frac{3 u^{2}}{2 V^{2}} \frac{\gamma}{\left(r+k^{2}\right)} \sum_{\mathbf{k}_{1}, \mathbf{k}_{2}} \frac{1}{\left(r+k_{1}^{2}\right)\left(r+k_{2}^{2}\right)\left(r+\left|\mathbf{k}_{1}+\mathbf{k}_{2}-\mathbf{k}\right|^{2}\right)} \exp \left\{\left[-\gamma\left(3 r+k_{1}^{2}+k_{2}^{2}+\left|\mathbf{k}_{1}+\mathbf{k}_{2}-\mathbf{k}\right|^{2}\right)\right] t\right\} \\
& G(\mathbf{k}, z)=\frac{1}{G_{0}^{-1}(\mathbf{k}, z)+\Sigma(\mathbf{k}, z)} \\
& \Sigma(\mathbf{k}, z)=c_{1}^{-1}(\mathbf{k})-\frac{3 u^{2}}{2 V^{2}} \sum_{\mathbf{k}_{1}, \mathbf{k}_{2}} \frac{1}{\left(r+k_{1}^{2}\right)\left(r+k_{2}^{2}\right)\left(r+\left|\mathbf{k}_{1}+\mathbf{k}_{2}-\mathbf{k}\right|^{2}\right)} \frac{z}{\left[z+\gamma\left(3 r+k_{1}^{2}+k_{2}^{2}+\left|\mathbf{k}_{1}+\mathbf{k}_{2}-\mathbf{k}\right|^{2}\right)\right]}
\end{aligned}
$$

where $G_{0}(\mathbf{k}, z)=1 /\left[z / \gamma+\left(r+k^{2}\right)\right]$, and $C_{1}(\mathbf{k})$ is the perturbed part of $C(\mathbf{k})=\left\langle|\sigma(\mathbf{k})|^{2}\right\rangle_{0}$ [cf. Eq. (3.11) of paper I]. Let us mention briefly some features of these results. As can be seen from Eq. (18), the memory function (and thus the correlation function) is neither even nor odd. This is not in agreement with the general theorem that states that any autocorrelation function of some physical property is even. This is not difficult to understand, if we observe that the Fokker-Planck operator defined by Eq. (4) does not have any symmetry under time reversal. In other words, $D$ is not affected as $t \rightarrow-t$, whatever the signature $( \pm 1)$ of $\sigma(\mathbf{k})$ under this transformation. The breaking of this symmetry is a consequence of the pure phenomenological approach used here. Another consequence of the phenomenology is that $\Phi(\mathbf{k}, t)$ has an exponential decay, which is not allowed by a theorem formulated by Lee. ${ }^{9}$ Although phenomenological models do not satisfy general theorems of the theory of correlation functions, they have been very successful in describing the critical dynamics of the system as it approaches the critical point.

Now, we will consider the critical dynamics aspects of the system according to Eqs. (19) and (20). For this end, we will review very briefly the main ideas of the dynamical renormalization-group transformations. The renormalization group consists of a set of transformations in which, first, the length, wave vector, spin density, and frequency are rescaled, and, second, the internal wave vectors [ $\mathbf{k}_{1}$ and $\mathbf{k}_{2}$ in Eq. (20)] are summed in the domain of $b^{-1} \Lambda<k_{1}<\Lambda$, where $b>1$, and $\Lambda$ is a cutoff wave vector. The effect of these transformations is to obtain a new GL Hamiltonian and a Fokker-Planck operator with renormalized constants $r, u$, and $\gamma$. Thus, we may repeatedly apply the renormalization-group transformation $l$ times to obtain renormalized constants $r_{l}, u_{l}$, and $\gamma_{l}$, which are defined by ${ }^{6(b),(c)}$

$$
\begin{aligned}
& r_{l}=\lim _{\substack{\mathbf{k} \rightarrow 0 \\
z \rightarrow 0}}\left(R_{b}^{s}\right)^{l}\left[G_{0}^{-1}(\mathbf{k}, z)+\Sigma_{l}(\mathbf{k}, z)\right], \\
& \gamma_{l}^{-1}=\lim _{\substack{\mathbf{k} \rightarrow 0 \\
z \rightarrow 0}} \frac{\partial}{\partial z}\left\{\left(R_{b}^{s}\right)^{l}\left[G_{0}^{-1}(\mathbf{k}, z)+\Sigma_{l}(\mathbf{k}, z)\right]\right\},
\end{aligned}
$$

where the effect of the transformation $R_{b}^{s}$ is to rescale the properties specified previously, and the subscript $l$ in the self-energy indicates that the sums are taken in the shell $\Lambda / b^{l}<k_{1}<\Lambda$. Let us denote by $\Sigma^{p}(\mathbf{k}, z)$ the self-energy derived by previous works. ${ }^{6}$ The term proportional to $u^{2}$ of its expression can be obtained from Eq. (20) by substi- tuting $z$ in the denominator of the second term by $\gamma\left(3 r+k_{1}^{2}+k_{2}^{2}+\left|\mathbf{k}_{1}+\mathbf{k}_{2}-\mathbf{k}\right|^{2}\right)$ and by deleting the term proportional to $u^{2}$ in $C_{1}^{-1}(\mathbf{k})$. Both expressions are exactly the same in the limit $z \rightarrow 0$, whatever the value of $\mathbf{k}$. Therefore the recursion relation for $r_{l}$, produced by Eqs. (19) and (21), would have no difference from the conventional one. This is also true for the recursion relation for $\gamma_{l}$. We can easily see this if we note that $\lim _{z \rightarrow 0} \partial \Sigma^{p}(\mathbf{k}, z) / \partial z=\lim _{z \rightarrow 0} \partial \Sigma(\mathbf{k}, z) / \partial z$ (this identity is no longer true for all $z \neq 0$ ). The determination of the fixed points and the (static and dynamic) critical exponents will be omitted here (see Ref. 6), since it is not important for our purposes.

The simplicity, but remarkable effectiveness, of the above-given renormalization-group analysis shows us how the difficulty arising from the differences between the Green's function, derived by using the POM and Feynman diagram method, can be overcome. In paper II this difference was attributed to the fact that the initial conditions (when the system is prepared) have been established at different instants (here, it is $t=0$, whereas in Ref. 6 it is $t=-\infty)$. Thus our critical dynamics, considerations also show that the behavior of the system near the critical point is always the same, independent of the instant it was prepared.

This work was supported by CNPq-Conselho Nacional de Desenvolvimento Científico e Tecnológico-Brazil under Grant No. 200471/88.0.

\section{APPENDIX A}

Using the definition of the projection operator [cf. Eq. (7)], we have shown that

$$
\begin{aligned}
& \int d \Gamma[P D P \rho(t)] \sigma(\mathbf{k}) \\
& \quad=\int d \Gamma\left[\rho_{0} \int d \Gamma D P \rho(t)\right. \\
& \left.\quad+\frac{\sigma(-\mathbf{k}) \rho_{0}}{\left\langle|\sigma(\mathbf{k})|^{2}\right\rangle_{0}} \int d \Gamma[D P \rho(t)] \sigma(\mathbf{k})\right] .
\end{aligned}
$$

Since $\int d \Gamma \rho_{0} \sigma(\mathbf{k})=0$, and by the definition $\left\langle|\sigma(\mathbf{k})|^{2}\right\rangle_{0}=\int d \Gamma \rho_{0} \sigma(\mathbf{k}) \sigma(-\mathbf{k})$, Eq. (A1) becomes 


$$
\begin{aligned}
\int d & \Gamma[P D P \rho(t)] \sigma(\mathbf{k}) \\
= & \int d \Gamma[D P \rho(t)] \sigma(\mathbf{k}) \\
= & \int d \Gamma\left[D \left[\rho_{0} \int d \Gamma \rho(t)\right.\right. \\
& \left.\left.\quad+\frac{\sigma(-\mathbf{k}) \rho_{0}}{\left\langle|\sigma(\mathbf{k})|^{2}\right\rangle_{0}} \int d \Gamma \rho(t) \sigma(\mathbf{k})\right] \sigma(\mathbf{k})\right] .
\end{aligned}
$$

Now, $D\left[\sigma(-\mathbf{k}) \rho_{0}\right]=-\gamma \rho_{0} \partial H / \partial \sigma(\mathbf{k})[$ cf. Eq. (3.4) of paper I], and by definition $C(\mathbf{k}, t)=\int d \Gamma \rho(t) \sigma(\mathbf{k})$.
Then, Eq. (A2) may be rewritten as

$$
\begin{aligned}
& \int d \Gamma[P D P \rho(t)] \sigma(\mathbf{k}) \\
& \quad=-\frac{\gamma}{\left\langle|\sigma(\mathbf{k})|^{2}\right\rangle_{0}} C(\mathbf{k}, t) \int d \Gamma \rho_{0} \frac{\partial H}{\partial \sigma(\mathbf{k})} \sigma(\mathbf{k}) .
\end{aligned}
$$

By using the identity

$$
\left\langle\frac{\partial H}{\partial \sigma(\mathbf{k})} F\right\rangle_{0}=\left\langle\frac{\partial F}{\partial \sigma(\mathbf{k})}\right\rangle
$$

we finally obtain Eq. (12).
*Present address: Department of Theoretical Physics, University of Manchester, Manchester M13 9PL, England.

${ }^{1}$ H. Mori, Prog. Theor. Phys. 33, 423 (1965).

${ }^{2}$ R. Zwanzig, J. Chem. Phys. 33, 1338 (1960).

${ }^{3}$ For a more comprehensive review of Refs. 1 and 2, see P. Grigolini, Adv. Chem. Phys. 62, 1 (1985).

${ }^{4}$ E. Sardella and A. F. S. Moreira, Prog. Theor. Phys. 82, 911 (1989).

${ }^{5}$ E. Sardella dn A. F. S. Moreira (unpublished).

${ }^{6}$ (a) S.-K. Ma, Modern Theory of Critical Phenomena (Benjamin,
New York, 1976); (b) B. I. Halperin, P. C. Hohenberg, and S.-K. Ma, Phys. Rev. B 10, 139 (1974); (c) P. C. Hohenberg and I. Halperin, Rev. Mod. Phys. 49, 435 (1977).

${ }^{7}$ M. Bixon and R. Zwanzig, J. Stat. Phys. 3, 245 (1971).

${ }^{8}$ The expressions for $\Sigma(\mathbf{k}, z)$ and $G_{0}(\mathbf{k}, z)$ differ by a minus sign from those of paper I, because we used the relationship between the Green's function and the correlation function as $G(\mathbf{k}, z)=z C(\mathbf{k}, z)-C(\mathbf{k})$, instead of the standard one, $G(\mathbf{k}, z)=C(\mathbf{k})-z C(\mathbf{k}, z)$.

${ }^{9}$ M. H. Lee, Phys. Rev. Lett. 51, 1227 (1983). 